\title{
Oxidative stress and cellular immunity in patients with recurrent aphthous ulcers
}

\author{
E. Avci ${ }^{1}$, Z.Z. Akarslan ${ }^{2}$, H. Erten ${ }^{3}$ and S. Coskun-Cevher ${ }^{4}$ \\ ${ }^{1}$ Department of Molecular Biology and Genetics/Biochemistry, Faculty of Science and Arts, Hitit University, Corum, Turkey \\ ${ }^{2}$ Department of Dentomaxillofacial Radiology, Faculty of Dentistry, Gazi University, Ankara, Turkey \\ ${ }^{3}$ Department of Operative Dentistry, Faculty of Dentistry, Gazi University, Ankara, Turkey \\ ${ }^{4}$ Department of Biology, Faculty of Science and Arts, Gazi University, Ankara, Turkey
}

\begin{abstract}
Recurrent aphthous ulcer (RAU) is an inflammatory condition of the oral mucosa characterized by painful, well-circumscribed, single or multiple round or ovoid ulcerations. The exact etiologic factor(s) of these ulcerations are not yet understood. The objective of this study was to evaluate inflammatory processes and free radical metabolism of 25 patients with RAUs compared to 25 healthy controls. The levels of malondialdehyde (MDA) and glutathione (GSH) were determined by high-performance liquid chromatography. Tumor necrosis factor-alpha (TNF- $\alpha$ ), interleukin-2 (IL-2), IL-10, and IL-12 were determined by ELISA. Nitric oxide (NO), myeloperoxidase (MPO), total antioxidant status (TAS), and total oxidant status (TOS) levels were measured spectroscopically in serum. The levels of MDA, GSH, TNF- $\alpha, \mathrm{IL}-2, \mathrm{IL}-12$, MPO, and TOS, and oxidative stress index (OSI) were higher, and the levels of NO, IL-10, and TAS were lower in patients with RAU than in controls. Statistical analysis showed that GSH, TNF- $\alpha$, IL-2, IL-10, and OSI differed significantly in patients with RAU compared to controls. These parameters have important roles in oxidant/antioxidant defense.
\end{abstract}

Key words: Recurrent aphthous ulcer; Oxidative stress; Cytokines

\section{Introduction}

Recurrent aphthous ulcer (RAU) is a lesion of the oral mucosa that is characterized by multiple recurrent, small, round or ovoid ulcers with circumscribed margins, erythematous haloes and yellow or gray floors (1-3). Although RAU lesions are very commonly observed in the oral mucosa, the exact etiological factor(s) are not yet understood. Contributing factors include allergies, genetic predisposition, hormonal influences, hematologic abnormalities, immunologic factors, infectious agents, nutritional deficiencies, smoking cessation, stress, and trauma. These factors have a direct or indirect potential for disturbing the equilibrium between oxidant and antioxidant systems in humans. The formation of free radicals could be accelerated and toxic reactions could occur once this equilibrium is disturbed $(4,5)$.

Reactive oxygen radicals are the most common free radicals present in biological systems. Oxidative stress occurs when intracellular concentrations of free radicals increase over the physiological values, which results in cell damage by lipid peroxidation, DNA and protein damage, enzyme oxidation, and stimulation of proinflammatory cytokine release $(2,6,7)$. Mammalian cells have developed enzymatic and nonenzymatic antioxidant defense systems to prevent oxidative damage caused by oxidative stress (8).

Malondialdehyde (MDA) is a stable end product of the peroxidation of membrane lipids by reactive oxygen species (ROS), and, thus, it is used as an indicator of increased lipid peroxidation. Interactions between MDA and membrane components result in disturbed structure and function of cell membranes (9). Nitric oxide (NO) is a freely diffusible intracellular and intercellular messenger that is synthesized from L-arginine by the family of NO synthase enzymes in mammalian cells. NO is both a gas and a free radical that reacts with many biological molecules. Increased production of NO during several inflammatory processes has been proposed (10). Another parameter related to oxidative stress is myeloperoxidase (MPO), which is a heme-containing enzyme found in azurophilic granules of human neutrophils (11). MPO is a heme enzyme that uses the oxidizing potential of superoxide and hydrogen peroxide to convert chloride ion to hypochlorous acid and other ROS (12). MPO, which is released during inflammation, is an oxidative enzyme

Correspondence: E. Avci, Department of Molecular Biology and Genetics/Biochemistry, Faculty of Science and Arts, Hitit University, Corum, Turkey. Fax: +90-364-227-7005. E-mail: emreavci@hitit.edu.tr and/or avci.emre@yahoo.com 
present in phagocytes. MPO could be a key element responsible for oxidative damage in the human artery wall (13). Glutathione (GSH) is an antioxidant whose role is to prevent damage caused by ROS (14). When oxidative stress is present, GSH is converted to its oxidized form of glutathione disulfide. The ratio of reduced $\mathrm{GSH}$ to oxidized glutathione within cells is often used as a measure of cellular toxicity. Therefore, an increase in glutathione disulfide is considered indicative of oxidative stress (15).

The aphthous process is believed to be initiated by stimulation of the mucosal keratinocytes by a currently unknown antigen, leading to T-lymphocyte stimulation and the liberation of cytokines and various interleukins. $\mathrm{TNF}-\alpha$ is a major inflammatory cytokine that has a chemotactic effect on neutrophils, thus driving acute inflammation and expression of major histocompatibility complexes. Changes in the TNF- $\alpha$ level and in IL-2, IL-4, $\mathrm{IL}-5$, and IL-10 levels in RAU have been postulated $(5,16)$.

Although there are reports that have partially investigated these parameters in patients with RAU, to the best of our knowledge, no study has evaluated all of these parameters in one trial, which could aid in determining a relationship between the oxidant/antioxidant status of serum and the inflammatory condition. This is the first study to assay MDA and GSH parameters in the blood samples of RAU patients using the highperformance liquid chromatography (HPLC) method. Therefore, the aim of this study was to assess inflammatory processes and free radical metabolism of patients with RAU.

\section{Material and Methods}

\section{Subjects}

Ethical approval was obtained from the Ethics Committee of the Gazi University (No. 09.06.2009/71), and written consent was given by all participants according to the Helsinki Declaration. A power analysis was performed before the study to determine the minimum patient and control numbers. According to this analysis, the number of patients and controls was found to be 21 for each group. Therefore, 25 subjects were planned in each group.

The study subjects were patients attending a dental school for treatment, and were asked to participate after a routine dental examination and an explanation of the study. A total of 26 females and 24 males from 19-40 years of age (mean $\pm S D, 28.9 \pm 3.8$ ) participated. The patient group was selected following a detailed case history and clinical examination. Participants with indications or recent history of acute inflammation and/or indications or history of systemic diseases such as respiratory, cardiovascular, endocrine, Behcet's, Crohn's and celiac diseases, anemia, or vitamin deficiency were not included. In addition, all participants were not currently taking any medications, were nonsmokers (never smoked), and free of any periodontal disease (all probing depths $\leq 3 \mathrm{~mm})(17,18)$. The patient group $(n=25)$ presented acute, minor RAU lesions diagnosed by a dentist during the collection of serum samples and had experienced oral ulcer attacks at least three times a year. The control group $(n=25)$ was selected from participants who had the same characteristics as the patients and were matched for age and gender. They did not have any history and clinical signs of RAU.

\section{Blood samples}

Blood samples were collected at the same time the dental examination of each patient was performed. The blood samples were prepared as appropriate and stored at $-80^{\circ} \mathrm{C}$ until biochemical analyses were performed.

\section{Measurement of MDA}

For the determination of MDA levels, a derivatization step was used, in which protein-bound MDA was hydrolyzed $\left(60 \mathrm{~min}\right.$ at $\left.95^{\circ} \mathrm{C}\right)$ and converted into a fluorescent probe. The fluorescent probe was cooled to $2-8^{\circ} \mathrm{C}$, centrifuged at $10,000 \mathrm{~g}$ for $5 \mathrm{~min}$, mixed with a reaction solution and injected into the HPLC system (Agilent Technologies 1200 series, USA; kits: Immuchrom $\mathrm{GmbH}$, Germany). The isocratic separation via HPLC at $30^{\circ} \mathrm{C}$, using a reversed-phase column (Bischoff Prontosil Eurobond, $5 \mu \mathrm{m}, 125 \mathrm{~mm} \times 4 \mathrm{~mm}$; Germany), lasted for $4 \mathrm{~min}$ for each sample. The chromatograms were recorded by a fluorescence detector. Quantification was performed with the calibrator from the kit. The concentration was calculated via integration of the peak heights using the following equation:

$$
\begin{aligned}
& \text { conc }_{\text {sample }}=(\text { peak height } \\
& / \text { peatient } \\
&\left.\times \text { conc }_{\text {calibrator }}\right) \\
& \text { height } \\
& \text { calibrator }
\end{aligned}
$$

\section{Measurement of GSH}

The levels of GSH were measured. During the derivatization reaction, glutathione was converted into a fluorescent probe. A subsequent precipitation step removed high molecular weight substances. After centrifugation at $10,000 \mathrm{~g}$ for $5 \mathrm{~min}$, the fluorescent probe was cooled to $2-8^{\circ} \mathrm{C}$ and injected into the HPLC system. The isocratic separation via HPLC at $30^{\circ} \mathrm{C}$ was performed with a reversed-phase column ( $\mathrm{MZ}$ Inertsil ODS, $5 \mu \mathrm{m}, 125 \mathrm{~mm} \times 4 \mathrm{~mm}$ ) in 2 runs. Each of these runs lasted $4 \mathrm{~min}$. The chromatograms were recorded by a fluorescence detector. The quantification was performed with the delivered EDTA-blood calibrator; the concentration was calculated using the internal standard (IS) method. GSH was calculated by the following equation: 


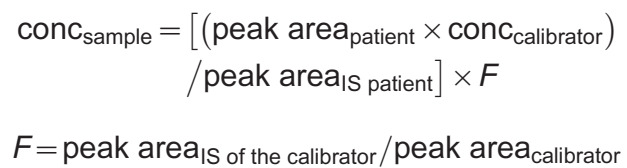

\section{Measurement of NO}

The NO values were given as the sum of nitrite and nitrate, which are the stable end products of NO. The nitrate levels in the samples were spectrophotometrically determined based on the reduction of nitrate to nitrite by $\mathrm{VaCl}_{3}$. Nitrite levels were measured by the Griess reaction. Measurement of NO was done by a modified spectrophotometric method used by Green et al. (19). Sodium nitrite and nitrate solutions $(1,10,50$, and $100 \mu \mathrm{M})$ were used as standards (19).

\section{Measurement of MPO}

MPO activity was assessed by measuring the $\mathrm{H}_{2} \mathrm{O}_{2}$ dependent oxidation of 0 -dianisidine. The MPO activities in the samples were determined with a modified spectrophotometric method (Shimadzu UV mini-1240, Japan) used by Colowick and Kaplan (20). One unit of enzymatic activity was defined as the amount of MPO that caused a change in the absorbance of $1.0 / \mathrm{min}$ at $410 \mathrm{~nm}$ at $37^{\circ} \mathrm{C}$.

\section{Measurement of TNF- $\alpha$ and interleukins (IL-2, IL-10, and IL-12)}

TNF- $\alpha$, IL-2, IL-10, and IL-12 levels were determined by enzyme-linked immunosorbent assay (ELISA; ELX 800 Reader, BioTek, USA). The following ELISAs (Instant ELISA, Bender MedSystems Diagnostic, eBioscience, Austria) were employed for the measurement of each parameter: human TNF- $\alpha$ (kit BMS223INST); human IL-2 (kit BMS221INST); IL-10 (kit BMS215INST), and IL-12 (high sensitivity kit BMS238HS).

\section{Measurement of total antioxidant status (TAS)}

Serum TAS was determined using a novel automated colorimetric measurement method developed by Erel (21). In this method, a hydroxyl radical is produced by the Fenton reaction and reacts with the colorless substrate $o$ dianisidine to produce the bright yellowish-brown dianisyl radical. The results are reported as micromol Trolox equivalents per liter ( $\mu \mathrm{mol}$ Trolox Eq/L).

\section{Measurement of total oxidant status (TOS)}

Serum TOS was determined using a novel automated colorimetric measurement method developed by Erel (22). In this method, the oxidants that were present in the sample oxidized the ferrous ion substrate o-dianisidine complex to the ferric ion. This reaction was enhanced by the glycerol molecules present in the reaction medium. The ferric ion produced a colored complex with xylenol orange in the acidic medium. Subsequently, the color intensity, related to the total amount of the oxidant molecules, was spectrophotometrically measured (Shimadzu UV mini1240). This assay was calibrated with hydrogen peroxide, and the results are reported as $\mu \mathrm{mol} \mathrm{H}_{2} \mathrm{O}_{2} \mathrm{Eq} / \mathrm{L}$.

\section{Determination of oxidative stress index (OSI)}

The ratio of TOS to TAS represents the OSI, an indicator of the degree of oxidative stress. The OSI value is calculated using the following formula:

$$
\begin{aligned}
\text { OSI }(\text { arbitrary unit })= & \operatorname{TOS}\left(\mu \mathrm{mol} \mathrm{H}_{2} \mathrm{O}_{2} \mathrm{Eq} / \mathrm{L}\right) \\
& / \operatorname{TAS}(\mu \mathrm{mol} \text { Trolox Eq } / \mathrm{L}) \times 100
\end{aligned}
$$

\section{Statistical analysis}

All data are reported as means $\pm S D$. All statistical analyses were performed using the SPSS version 15.0 statistical software package (SPSS Inc., USA). The data were analyzed with the Mann-Whitney U-test, the Levene test, and the $t$-test for equality of means of independent groups. The level of significance was set at $\mathrm{P}<0.05$.

\section{Results}

MDA $(P=0.596)$, GSH $(P=0.002)$, MPO $(P=0.571)$, TNF- $\alpha(P=0.004), \quad I L-2 \quad(P=0.001), \quad I L-12 \quad(P=0.384)$, TOS $(P=0.198)$, and OSI $(P=0.006)$ levels increased in the RAU group compared to the control group. On the other hand, $\mathrm{NO}(\mathrm{P}=0.089), \mathrm{IL}-10(\mathrm{P}=0.001)$ and TAS $(P=0.304)$ levels decreased in the RAU group compared to the control group. Statistical significance was only observed in GSH, TNF- $\alpha$, IL-2, IL-10, and OSI levels $(P<0.05)$. All parameters evaluated of RAU patients and healthy controls are summarized in Table 1.

\section{Discussion}

The role of antioxidants in the maintenance of health and chemoprevention of disorders and diseases has received great attention (23). A possible relationship has been suggested between inflammatory processes and free radical metabolism. Immune defects can disturb the oxidant/antioxidant balance of the organism and can accelerate the formation of free radicals. The cytotoxic effects of free radicals lead to cell damage and increase oxidative stress. Enzymes of the oxidative system may be induced by free radicals produced as a result of the infiltration and activation of lymphocytes. Lih-Brody et al. (24) found increased oxidative stress and decreased antioxidant defense in the mucosa of patients with inflammatory bowel disease, and Kokcam and Naziroglu (25) reported a potential role of increased lipid peroxidation and decreased antioxidants in psoriasis. As inflammatory mechanisms and oxidative stress have also been suggested in the pathogenesis of RAU, these parameters 
Table 1. Parameters evaluated in patients with RAU and healthy controls.

\begin{tabular}{lcc}
\hline Parameters & $\begin{array}{c}\text { Healthy controls } \\
(\mathrm{n}=25)\end{array}$ & $\begin{array}{c}\text { Patients with } \\
\text { RAU }(\mathrm{n}=25)\end{array}$ \\
\hline $\mathrm{MDA}(\mu \mathrm{mol} / \mathrm{L})$ & $0.76 \pm 0.20$ & $1.20 \pm 0.78$ \\
$\mathrm{GSH}(\mu \mathrm{mol} / \mathrm{L})$ & $684.20 \pm 63.60$ & $864.26 \pm 102.04^{*}$ \\
$\mathrm{NO}(\mu \mathrm{mol} / \mathrm{L})$ & $19.84 \pm 4.96$ & $16.41 \pm 2.32$ \\
$\mathrm{MPO}(\mathrm{U} / \mathrm{mg}$ protein $)$ & $17.51 \pm 3.88$ & $18.74 \pm 3.66$ \\
$\mathrm{TNF}-\alpha(\mathrm{pg} / \mathrm{mL})$ & $3.45 \pm 1.01$ & $5.26 \pm 1.21^{*}$ \\
$\mathrm{IL}-2(\mathrm{pg} / \mathrm{mL})$ & $1.94 \pm 0.72$ & $3.97 \pm 0.38^{*}$ \\
$\mathrm{IL}-10(\mathrm{pg} / \mathrm{mL})$ & $1.56 \pm 0.29$ & $0.89 \pm 0.08^{*}$ \\
$\mathrm{IL}-12(\mathrm{pg} / \mathrm{mL})$ & $24.65 \pm 4.67$ & $27.56 \pm 4.92$ \\
$\mathrm{TAS}(\mu \mathrm{mol} \mathrm{Trolox} \mathrm{Eq} / \mathrm{L})$ & $2.15 \pm 0.32$ & $2.05 \pm 0.38$ \\
TOS $\left(\mu \mathrm{mol} \mathrm{H} \mathrm{O}_{2} \mathrm{Eq} / \mathrm{L}\right)$ & $3.54 \pm 0.67$ & $3.97 \pm 1.34$ \\
OSI $(\mathrm{arbitrary}$ units $)$ & $0.016 \pm 0.01$ & $0.019 \pm 0.03^{*}$ \\
\hline
\end{tabular}

RAU: recurrent aphthous ulcer; MDA: malondialdehyde; GSH: glutathione; NO: nitric oxide; MPO: myeloperoxidase; TNF- $\alpha$ : tumor necrosis factor-alpha; IL: interleukin; TAS: total antioxidant status; TOS: total oxidant status; OSI: oxidative stress index. ${ }^{*} \mathrm{P}<0.05$, compared to control (Mann-Whitney U-test, Levene test, and $t$-test).

were investigated in the present study. In general, we have reached similar conclusions in previous studies, but there are some inconsistencies. Differences in the results may be related to the notion that RAU has more than one etiologic factor. Many factors, such as allergies, genetic predisposition, hormonal influences, hematologic abnormalities, immunologic factors, infectious agents, nutritional deficiencies, smoking cessation, stress, and trauma $(4,5)$ are thought to be responsible for the occurrence of RAU.

In the present study, we investigated whether there were any changes in all parameters in blood samples of patients with RAU compared with those of healthy controls. In this study, MDA levels were found to be higher, although not statistically significant, in the serum of RAU patients compared with the control group. Increased MDA levels may indicate that peroxidation reactions are activated due to an impaired antioxidant defense system in the extracellular fluid, which may be an indication of oxidative stress derived from inflammation. Some authors have reported that MDA levels in the plasma of RAU patients were higher than in the control group $(26,27)$. Our findings included high levels of GSH in blood samples, which support the effect of oxidative stress as demonstrated by the increased TOS in RAU. Previous studies that evaluated glutathione peroxidase activity reported results inconsistent with those of this investigation. Cimen et al. (26) reported a decrease in glutathione peroxidase in erythrocytes. Karincaoglu et al. (28) reported decreased SOD and CAT activity in plasma of patients with RAU. Momen-Beitollahi et al. (2) reported no significant change in the glutathione peroxidase activity in erythrocytes. These differences may be associated with the use of various methods, as HPLC and blood samples were used in the present study, and/or the genetic divergence of each population. Additionally, the antioxidant composition and capacity may vary with dietary and nutrient deficiency. NO levels were found to decrease in serum of RAU patients compared with controls, but the change was not statistically significant. This finding indicated that NO does not appear to play a major role in the formation of RAU. Gunduz et al. (29) reported no significant change in plasma $\mathrm{NO}$ levels in RAU and Behcet's disease. Ozkan et al. (30) reported no significant difference between the serum NO levels in RAU and healthy patients, but reported a significant difference among patients with Behcet's disease.

Given the results obtained from our study, we suggest that abnormalities of the cytokine mechanism (elevated TNF- $\alpha$, IL-2, IL-12, and decreased IL-10 levels) may have accelerated lipid peroxidation and lead to an increase in oxidative stress in the serum. Simultaneously, the antioxidant mechanisms of serum are decreased. Due to low levels of IL-10, which functions to inhibit the release of cytokines, the inflammatory reaction may not be suppressed, and, hence, RAU may occur. Studies have indicated that T-cell immunity plays a role in the etiology of RAU (31-33). T-cell immunity reactions are described as $\mathrm{TH} 1$ and $\mathrm{TH} 2$ reactions. It is believed that IL-12 initiates $\mathrm{TH} 1$ reactions, and $\mathrm{IL}-10$ initiates $\mathrm{TH} 2$ reactions. As the $\mathrm{TH} 1$ reaction is dominant in RAU, the T cells are thought to be induced by elevated IL-12 levels or decreased IL-10 levels (34). Sun et al. (35) reported that the CD4 +, CD4 + $\mathrm{IL}-2 \mathrm{R}+$, and CD8 + IL-2R + cell counts and the serum level of IL-2 increased simultaneously in patients in the exacerbation stage of RAU. Those authors suggested that the markedly increased serum level of IL-2 may have resulted from secretion by the increased number of activated CD4 + cells and that the expression of IL-2R was upregulated by the serum level of IL-2 in patients with RAU. Buno et al. (36) reported elevated levels of IL-2 in lesioned mucosa and decreased levels of IL-10 mRNA in the acute RAU site. IL-10 typically stimulates epithelial proliferation in the healing process. Low levels in RAU may suggest a delay in epithelialization and prolongation of the duration of the lesions. As occurrence of RAU diminishes with advancing age, it is also of interest that whole blood assays reveal greater IL-10 production in the elderly compared with younger controls (37). Our results demonstrated an increase in IL-2 and IL-12 levels and a decrease in the IL-10 level in serum of patients with RAU. In addition, an approximately 1.5-fold increase in the level of TNF- $\alpha$ was present in RAU patients. Increased levels of TNF- $\alpha$ in serum of RAU patients were also reported by different authors $(5,38,39)$. This result emphasizes the proinflammatory effect of this cytokine in RAU. These results also favor an active, cell-mediated immune process, leading to the failure of the suppression of inflammation, thus, resulting in an aphthous ulcer. 
Serum TAS and TOS levels were evaluated in RAU patients in only a few studies $(3,39)$. Those investigations demonstrated that RAU patients had higher TOS and lower TAS levels than healthy controls. In our study, the TOS levels were found to be higher in the serum of patients with RAU than in controls, but the difference was not significant. The increase in the TOS indicated increased lipid peroxidation in serum, which indicated oxidative stress in these patients. Additionally, the TAS levels were found to be lower in serum of patients with RAU than in controls, but again, the difference was not significant. One may conclude that the decrease in the serum antioxidant capacity may have led to an increase in the TOS of serum and/or are due to low levels of antioxidant capacity, that is, the body could not sufficiently eradicate the harmful effects of the increased oxidative stress. The difference in serum TAS may be related to i) the increased sensitivity of human peripheral blood lymphocytes to agents such as $\mathrm{H}_{2} \mathrm{O}_{2}$, and the greater number of DNA strand breaks in those cells compared with other cell types (28), and/or ii) the increased production of ROS by peripheral blood neutrophils (40). The OSI detected in our study was higher in patients with RAU than in healthy controls indicating the exact degree

\section{References}

1. Caglayan F, Miloglu O, Altun O, Erel O, Yilmaz AB. Oxidative stress and myeloperoxidase levels in saliva of patients with recurrent aphthous stomatitis. Oral Dis 2008; 14: 700-704, doi: 10.1111/j.1601-0825.2008.01466.x.

2. Momen-Beitollahi J, Mansourian A, Momen-Heravi $F$, Amanlou M, Obradov S, Sahebjamee M. Assessment of salivary and serum antioxidant status in patients with recurrent aphthous stomatitis. Med Oral Patol Oral Cir Bucal 2010; 15: e557-e561, doi: 10.4317/medoral.15.e557.

3. Akoglu G, Metin A, Kilinc F, Pektas SD, Isikoglu S, Akbas A, et al. Total serum oxidant/antioxidant status and arylesterase activity in recurrent aphthous stomatitis. Ann Dermatol 2013; 25: 273-277, doi: 10.5021/ad.2013.25.3.273.

4. Boras VV, Lukac J, Brailo V, Picek P, Kordic D, Zilic IA. Salivary interleukin-6 and tumor necrosis factor-alpha in patients with recurrent aphthous ulceration. J Oral Pathol Med 2006; 35: 241-243, doi: 10.1111/j.1600-0714.2006. 00404.x.

5. Eguia-del Valle A, Martinez-Conde-Llamosas R, LopezVicente J, Uribarri-Etxebarria A, Aguirre-Urizar JM. Salivary levels of tumour necrosis factor-alpha in patients with recurrent aphthous stomatitis. Med Oral Patol Oral Cir Bucal 2011; 16: e33-e36.

6. Chapple IL. Role of free radicals and antioxidants in the pathogenesis of the inflammatory periodontal diseases. Clin Mol Pathol 1996; 49: M247-M255, doi: 10.1136/mp.49.5. M247.

7. Chapple IL, Brock G, Eftimiadi C, Matthews JB. Glutathione in gingival crevicular fluid and its relation to local antioxidant capacity in periodontal health and disease. Mol Pathol 2002; 55: 367-373, doi: 10.1136/mp.55.6.367. of imbalance of oxidative stress toward the oxidant status. In a previous study, it was reported that serum TAS was decreased in patients with RAU, and there was no significant difference between the serum total antioxidant status in the active and remission periods of the lesion (40). Although these findings do not allow the establishment of the exact role for ROS in the development of $R A U$, our results are consistent with the notion that oxidative stress plays a vital role in the pathophysiological component of the lesion.

In conclusion, the levels of parameters that differed between the study groups have important roles in oxidant/ antioxidant defense in patients with RAU compared with healthy controls. MDA, GSH, MPO, TNF- $\alpha$, IL-2, IL-12, TOS levels, and OSI were increased, and NO, IL-10, and TAS levels were decreased in RAU patients. Statistically significant differences indicated that GSH, TNF- $\alpha$, IL-2, IL-10, and OSI play important roles in the occurrence of RAU.

\section{Acknowledgments}

Research supported by the BAP project (\#03/201007) of the Gazi University, Turkey.

8. Raborn GW, Grace MG. Recurrent herpes simplex labialis: selected therapeutic options. J Can Dent Assoc 2003; 69 : 498-503.

9. Saral Y, Coskun BK, Ozturk P, Karatas F, Ayar A. Assessment of salivary and serum antioxidant vitamins and lipid peroxidation in patients with recurrent aphthous ulceration. Tohoku J Exp Med 2005; 206: 305-312, doi 10.1620/tjem.206.305.

10. Bosca L, Zeini M, Traves PG, Hortelano S. Nitric oxide and cell viability in inflammatory cells: a role for $\mathrm{NO}$ in macrophage function and fate. Toxicology 2005; 208: 249258, doi: 10.1016/j.tox.2004.11.035.

11. Kaner D, Bernimoulin JP, Kleber BM, Heizmann WR, Friedmann A. Gingival crevicular fluid levels of calprotectin and myeloperoxidase during therapy for generalized aggressive periodontitis. J Periodontal Res 2006; 41: 132139, doi: 10.1111/j.1600-0765.2005.00849.x.

12. Daugherty A, Dunn JL, Rateri DL, Heinecke JW. Myeloperoxidase, a catalyst for lipoprotein oxidation, is expressed in human atherosclerotic lesions. J Clin Invest 1994; 94: 437-444, doi: 10.1172/JCI117342.

13. Zouaoui BK, Moguilevsky N, Legssyer I, Babar S, Guillaume $M$, Delree $P$, et al. Oxidation of low density lipoproteins by myeloperoxidase at the surface of endothelial cells: an additional mechanism to subendothelium oxidation. Biochem Biophys Res Commun 2004; 325: 434-438, doi: 10.1016/j.bbrc.2004.10.049.

14. Pompella A, Visvikis A, Paolicchi A, De Tata V, Casini AF. The changing faces of glutathione, a cellular protagonist. Biochem Pharmacol 2003; 66: 1499-1503, doi: 10.1016/S0006-2952 (03)00504-5. 
15. Pastore A, Piemonte F, Locatelli M, Lo Russo A, Gaeta LM, Tozzi G, et al. Determination of blood total, reduced, and oxidized glutathione in pediatric subjects. Clin Chem 2001; 47: 1467-1469.

16. Scully C, Hodgson T. Recurrent oral ulceration: aphthouslike ulcers in periodic syndromes. Oral Surg Oral Med Oral Pathol Oral Radiol Endod 2008; 106: 845-852, doi: 10.1016/ j.tripleo.2008.07.014.

17. Guentsch A, Preshaw PM, Bremer-Streck S, Klinger G, Glockmann E, Sigusch BW. Lipid peroxidation and antioxidant activity in saliva of periodontitis patients: effect of smoking and periodontal treatment. Clin Oral Investig 2008; 12: 345-352, doi: 10.1007/s00784-008-0202-z.

18. Buduneli N, Kardesler L, Isik H, Willis CS III, Hawkins SI, Kinane DF, et al. Effects of smoking and gingival inflammation on salivary antioxidant capacity. J Clin Periodontol 2006; 33: 159-164, doi: 10.1111/j.1600-051X.2006.00892.x.

19. Green LC, Wagner DA, Glogowski J, Skipper PL, Wishnok JS, Tannenbaum SR. Analyses of nitrate, nitrite and [15N] nitrate in biological fluids. Anal Biochem 1982; 126: 131138.

20. Colowick SP, Kaplan SD (Editors). Myeloperoxidase activity. In: Methods in enzymology. Vol II. New York: Academic Press; 1955. p 769-770.

21. Erel O. A novel automated method to measure total antioxidant response against potent free radical reactions. Clin Biochem 2004; 37: 112-119, doi: 10.1016/j.clinbiochem. 2003.10.014.

22. Erel $\mathrm{O}$. A new automated colorimetric method for measuring total oxidant status. Clin Biochem 2005; 38: 1103-1111, doi: 10.1016/j.clinbiochem.2005.08.008.

23. Niki E. Antioxidant capacity: Which capacity and how to assess it? J Berry Res 2012; 1: 169-176.

24. Lih-Brody L, Powell SR, Collier KP, Reddy GM, Cerchia R, Kahn E, et al. Increased oxidative stress and decreased antioxidant defenses in mucosa of inflammatory bowel disease. Dig Dis Sci 1996; 41: 2078-2086, doi: 10.1007/BF0 2093613.

25. Kokcam I, Naziroglu M. Antioxidants and lipid peroxidation status in the blood of patients with psoriasis. Clin Chim Acta 1999; 289: 23-31, doi: 10.1016/S0009-8981(99)00150-3

26. Cimen MY, Kaya TI, Eskandari G, Tursen U, Ikizoglu G, Atik U. Oxidant/antioxidant status in patients with recurrent aphthous stomatitis. Clin Exp Dermatol 2003; 28: 647-650, doi: 10.1046/j.1365-2230.2003.01415.x.

27. Ozturk P, Belge KE, Ataseven A. Copper/zinc and copper/ selenium ratios, and oxidative stress as biochemical markers in recurrent aphthous stomatitis. J Trace Elem Med Biol 2013; 27: 312-316, doi: 10.1016/j.jtemb.2013.04.002.

28. Karincaoglu Y, Batcioglu K, Erdem T, Esrefoglu M, Genc M. The levels of plasma and salivary antioxidants in the patient with recurrent aphthous stomatitis. J Oral Pathol Med 2005; 34: 7-12, doi: 10.1111/j.1600-0714.2004.00253.x.
29. Gunduz K, Ozturk G, Sozmen EY. Erythrocyte superoxide dismutase, catalase activities and plasma nitrite and nitrate levels in patients with Behcet disease and recurrent aphthous stomatitis. Clin Exp Dermatol 2004; 29: 176-179, doi: 10.1111/j.1365-2230.2004.01488.x

30. Ozkan Y, Yardim-Akaydin S, Sepici A, Engin B, Sepici V, Simsek B. Assessment of homocysteine, neopterin and nitric oxide levels in Behcet's disease. Clin Chem Lab Med 2007; 45: 73-77, doi: 10.1515/CCLM.2007.018.

31. Stenman G, Heyden G. Premonitory stages of recurrent aphthous stomatitis. I. Histological and enzyme histochemical investigations. J Oral Pathol 1980; 9: 155-162, doi: 10 1111/j.1600-0714.1980.tb00372.x.

32. Savage NW, Seymour GJ, Kruger BJ. Expression of class I and class II major histocompatibility complex antigens on epithelial cells in recurrent aphthous stomatitis. J Oral Pathol 1986; 15: 191-195, doi: 10.1111/j.1600-0714.1986. tb00605.x.

33. Healy $\mathrm{CM}$, Thornhill $\mathrm{MH}$. Induction of adhesion molecule expression on blood vessels and keratinocytes in recurrent oral ulceration. J Oral Pathol Med 1999; 28: 5-11, doi: 10. 1111/j.1600-0714.1999.tb01986.x.

34. Koybasý S, Parlak AH. Reccurrent aphthous stomatitis. Turkiye Klinkleri J Med Sci 2006; 26: 319-329.

35. Sun A, Chu CT, Liu BY, Wang JT, Leu JS, Chiang CP. Expression of interleukin-2 receptor by activated peripheral blood lymphocytes upregulated by the plasma level of interleukin-2 in patients with recurrent aphthous ulcers. Proc Natl Sci Counc Repub China B 2000; 24: 116-122.

36. Buno IJ, Huff JC, Weston WL, Cook DT, Brice SL. Elevated levels of interferon gamma, tumor necrosis factor alpha, interleukins 2, 4, and 5, but not interleukin 10, are present in recurrent aphthous stomatitis. Arch Dermatol 1998; 134: 827-831

37. Cakman I, Rohwer J, Schutz RM, Kirchner H, Rink L. Dysregulation between $\mathrm{TH} 1$ and $\mathrm{TH} 2 \mathrm{~T}$ cell subpopulations in the elderly. Mech Ageing Dev 1996; 87: 197-209, doi: 10.1016/0047-6374(96)01708-3.

38. Taylor LJ, Bagg J, Walker DM, Peters TJ. Increased production of tumour necrosis factor by peripheral blood leukocytes in patients with recurrent oral aphthous ulceration. J Oral Pathol Med 1992; 21: 21-25, doi: 10.1111/j. 1600-0714.1992.tb00963.x.

39. Sepici-Dincel A, Ozkan Y, Yardim-Akaydin S, KaymakKaratas G, Onder M, Simsek B. The association between total antioxidant status and oxidative stress in Behcet's disease. Rheumatol Int 2006; 26: 1005-1009, doi: 10.1007/ s00296-006-0126-3.

40. Lewkowicz N, Lewkowicz P, Kurnatowska A, Banasik M, Glowacka E, Cedzynski M, et al. Innate immune system is implicated in recurrent aphthous ulcer pathogenesis. J Oral Pathol Med 2003; 32: 475-481, doi: 10.1034/j.1600-0714. 2003.00181.x. 